\title{
Metal mesh filters based on Ti, ITO and Cu thin films for terahertz waves
}

\author{
Y. Demirhan ${ }^{1} \cdot$ H. Alaboz ${ }^{1} \cdot$ L. Ozyuzer ${ }^{1} \cdot$ M. A. Nebioğlu ${ }^{2} \cdot$

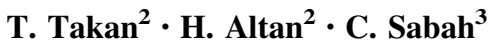

Received: 30 September 2015/ Accepted: 20 January 2016/Published online: 4 February 2016

(C) Springer Science+Business Media New York 2016

\begin{abstract}
In this study, we have investigated the spectral performance of resonant terahertz $(\mathrm{THz})$ bandpass filters which were produced from thin films with a metal-mesh shape. The aforementioned filters were fabricated from titanium, copper and indium tin oxide thin films on fused silica substrates by UV lithography with an array of cross-shaped apertures. Since the mesh period, cross-arm length and its width specify the spectral characteristics of the filters, we were able to reveal the performance of these filters experimentally using both a $\mathrm{THz}$ time domain spectrometer and a Fourier transform infrared spectrometer. A commercial electromagnetic simulation software, CST microwave studio, was used to verify the experimental data. The transmission of the filters are in the range 20-55\% at their relevant center frequencies. To our knowledge this study is the first to show that fabricated patterns based on ITO thin films can be used to filter $\mathrm{THz}$ radiation.
\end{abstract}

Keywords Terahertz radiation · Metal mesh filters · Frequency selective surfaces · Bandpass filters · Thin film fabrication

This article is part of the Topical Collection on TERA-MIR: Materials, Generation, Detection and Applications.

Guest Edited by Mauro F. Pereira, Anna Wojcik-Jedlinska, Renata Butkute, Trevor Benson, Marian Marciniak and Filip Todorov.

Y. Demirhan

yasemindemirhan@iyte.edu.tr

1 Department of Physics, Izmir Institute of Technology, 35430 Urla, Izmir, Turkey

2 Department of Physics, Middle East Technical University, 06800 Çankaya, Ankara, Turkey

3 Department of Electrical and Electronics Engineering, Middle East Technical University-Northern Cyprus Campus, Kalkanli, Guzelyurt, 99738 TRNC/Mersin 10, Turkey 


\section{Introduction}

In the electromagnetic spectrum, terahertz $(\mathrm{THz})$ radiation is in between microwaves and the far-IR. The frequencies in this region are in range of $0.1-10 \mathrm{THz}$ and the corresponding wavelengths are from 3 to $0.03 \mathrm{~mm}$. Due to its innovative applications in different areas such as non-destructive tests, military and civilian security, chemistry, medicine, biology, and others; terahertz range has attracted world-wide interest in the recent years (Tonouchi 2007; Ferguson and Zhang 2006; Kleine-Ostmann and Nagatsuma 2011; Siegel 2004). There are outstanding endeavors aiming to fill this vacancy in the spectrum (Ozyuzer et al. 2007; Kadowaki et al. 2010; Demirhan et al. 2015).

There are very few optical devices operating in the $\mathrm{THz}$ regime since the optical properties of most materials in this range of the electromagnetic spectrum are inadequate. While there is a high motivation to perform experiments in the $\mathrm{THz}$ frequency range, new sources, detectors and optical components are still needed. Recently, there have been many improvements in the development of THz sources (Turkoglu et al. 2012, 2013; Wang et al. 2009) and in the fabrication of THz detectors (Rice et al. 1994; Hu and Richards 1989; Du et al. 2008).

Moreover, many efforts to develop tunable $\mathrm{THz}$ filters have been impeded, where dispersive media to spatially filter out the unwanted components have been used (Melo et al. 2008; Porterfield et al. 1994; Ade et al. 2006; Tarasov et al. 2009; Park et al. 2010). In standard digital electronics, it is a usual procedure to design arbitrary and complicated filters enabling wireless devices which operate in the radio and microwave regions of the electromagnetic spectrum. When out-of-band interferences are blocked, the detection capabilities could be improved which leads to the transmission of only the desired spectral regions. When excess thermal radiation is blocked, the filtering procedure could be useful to flatten the output spectrum of a $\mathrm{THz}$ emitter and also the noise equivalent power of cryogenic bolometers (detectors) can be lowered. The analysis of physical mechanisms operating in the different filter structures were carried out within the common theoretical framework of extraordinary optical transmission (EOT) which was put forward almost 10 years ago. Surface electromagnetic modes play a key role in the emergence of the resonant transmission. It was also shown that, these modes are at the origin of the enhanced transmission from single apertures surrounded by periodic corrugations (Takan et al. 2015). Compared to these digital filters, the electromagnetic responses of structured conductive surfaces on which the metal-mesh filters are fabricated from are far more robust. Operation of these filters as high pass, low pass, bandpass filters is possible. By altering the geometry together with their dimensional parameters, the optical behavior of these filters can be altered (Melo et al. 2012). In several theoretical as well as experimental studies, the extraordinary transmission has been shown to play a vital role (Martin-Moreno et al. 2001; Ebbesen et al. 1998). The initial observation was made on silver, gold, and chromium films at optical frequencies (Kimand and Choi 2006).

Subsequent to the theoretical studies and experimental efforts, researchers started to investigate on producing several types of filters which show high-transmission peaks lying in the range $100 \mathrm{GHz}$ up to $14 \mathrm{THz}$ and with the property of bandpass widths between 13 up to $50 \%$ of the central frequency (Ma et al. 2009; Kaufmann et al. 2010; Winnewiser et al. 1999). In the bandpass geometry of the metal mesh filters, the conventional filter is the cross-shaped type in which the dimensions of the cross width $(\mathrm{J})$, length $(\mathrm{K})$, and periodicity $(\mathrm{G})$ determine the frequency profile. This type of metal mesh filters was initially introduced and investigated by Porterfield (Porterfield et al. 1994). The transmission 
Fig. 1 Sketch of a cross-shaped

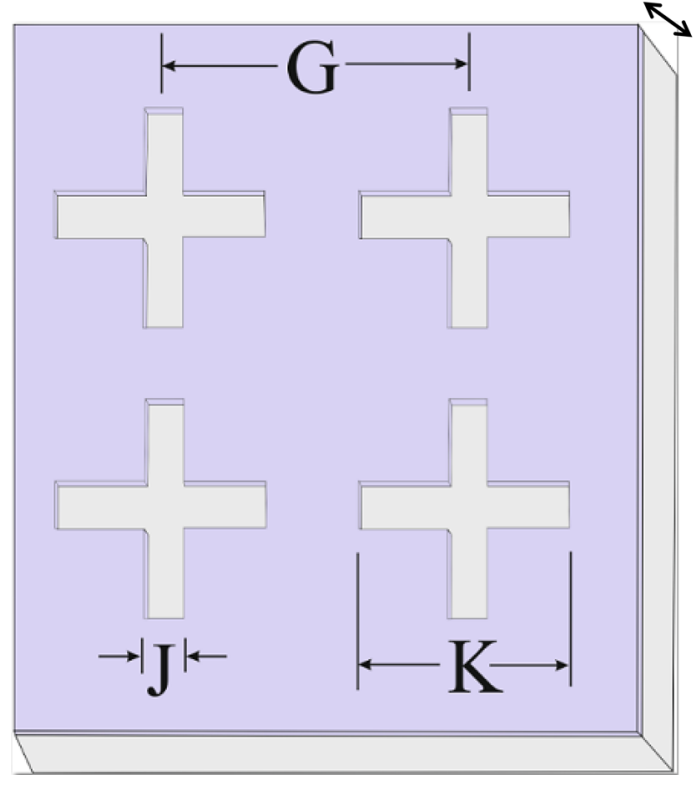

performance of the filter is determined by the design parameters when combined with the thickness $h$ of the material. In Fig. 1, the resonant metal-mesh filter design is presented.

In earlier studies, the resonant empirical formula for free standing filter structures is given by,

$$
\lambda_{\mathrm{r}}=1.8 \mathrm{~K}-1.35 \mathrm{~J}+0.2 \mathrm{G}
$$

where the wavelength of the cross-shaped filter can be calculated (Voisiat et al. 2011). The resonant frequency decreases by an increase in the length of the crosses $\mathrm{K}$ whereas when the width of the crosses $\mathrm{J}$ increases the bandwidth also increases and the resonant frequency slightly increases. There is a small decrease in the resonant frequency when the substrate thickness $h$ is increasing. On the other hand, the resonant frequency increases when the corners of the apertures are rounded.

In the present study, we have accomplished simulation and transmission measurements of cross shaped $\mathrm{Ti}$, ITO, Cu thin film bandpass $\mathrm{THz}$ filters on fused silica substrates with various dimensions and these filters are compact, present an easy and available fabrication process. The measurements have been discussed in the light of surface conductivity of deposited films.

\section{Design and fabrication of metal mesh filters}

Metal mesh filters are frequency selective surfaces that exhibit high transmission profiles at designed frequency and reject out of band radiation. In addition to that properties, the ideal bandpass filters (BPFs) must be vacuum compatible, vibration tolerant and cryogenically stable for most of $\mathrm{THz}$ applications. There are three main goals associated with filter design: increase the peak transmission, decrease the peak full width half maximum 
(FWHM) and decrease the out of band transmission. Decreasing out of band transmission is important for sensor technologies and it improves detection capabilities by blocking out of band interferences and allowing transmission of only the desired spectral region. It also improves signal to noise ratio (SNR) of a detector.

A priori to the experimental measurements, CST microwave studio simulations have been realized with the purpose of prescribing the transmission characteristics and also ensuring a design with optimum structure. We adopt the free standing filter design as shown in ref. (Porterfield et al. 1994) to the structures fabricated on fused silica substrates (for commercial use substrate is essential, thin metallic film is very sensitive to mechanical stresses). Unsurprisingly, the addition of the dielectric substrate affected the behavior of the resonant filters (Ma et al. 2009).

The numerical results for terahertz wave transmittance of the copper filter are shown in Fig. 2, the simulated results gives the terahertz wave transmittance peak of $82.5 \%$ at $0.333 \mathrm{THz}$ for $402(\mathrm{~K}) / 261(\mathrm{~J}) / 66(\mathrm{G}) \mu \mathrm{m}$ mesh parameter. In order to emphasize the effect of the material, we present the simulated results of ITO filter with same design parameters in Fig. 3. Resonant frequency was not affected by material type of the filter, however the
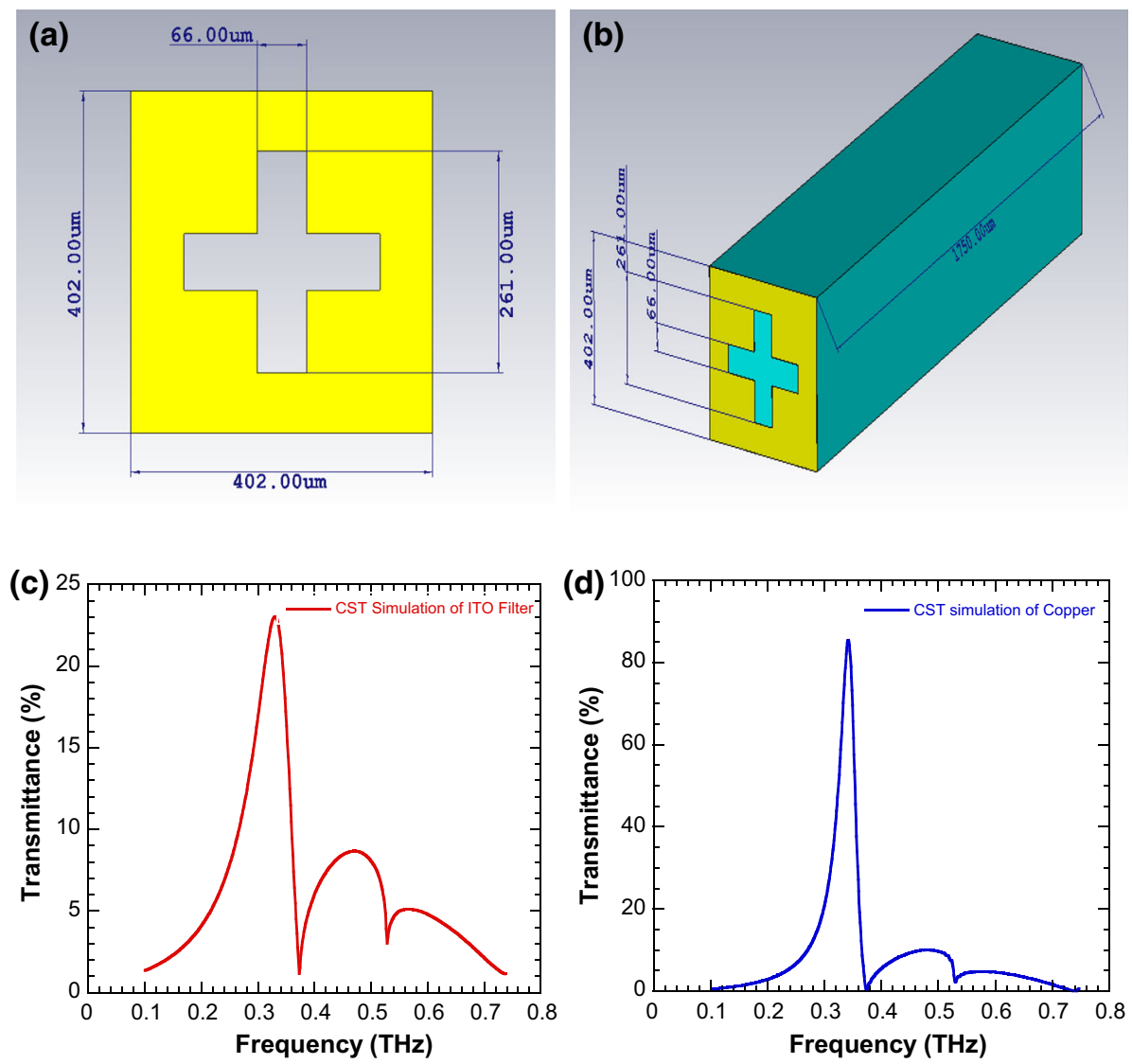

Fig. 2 a, b Design of metal mesh filters with dimension $\mathrm{G} / \mathrm{J} / \mathrm{K}, 402,261.66 \mu \mathrm{m}$ and substrate thickness of $1.75 \mathrm{~mm}$, respectively. c, d The simulated transmission spectrum of ITO and Cu metal mesh filters with designed parameters 

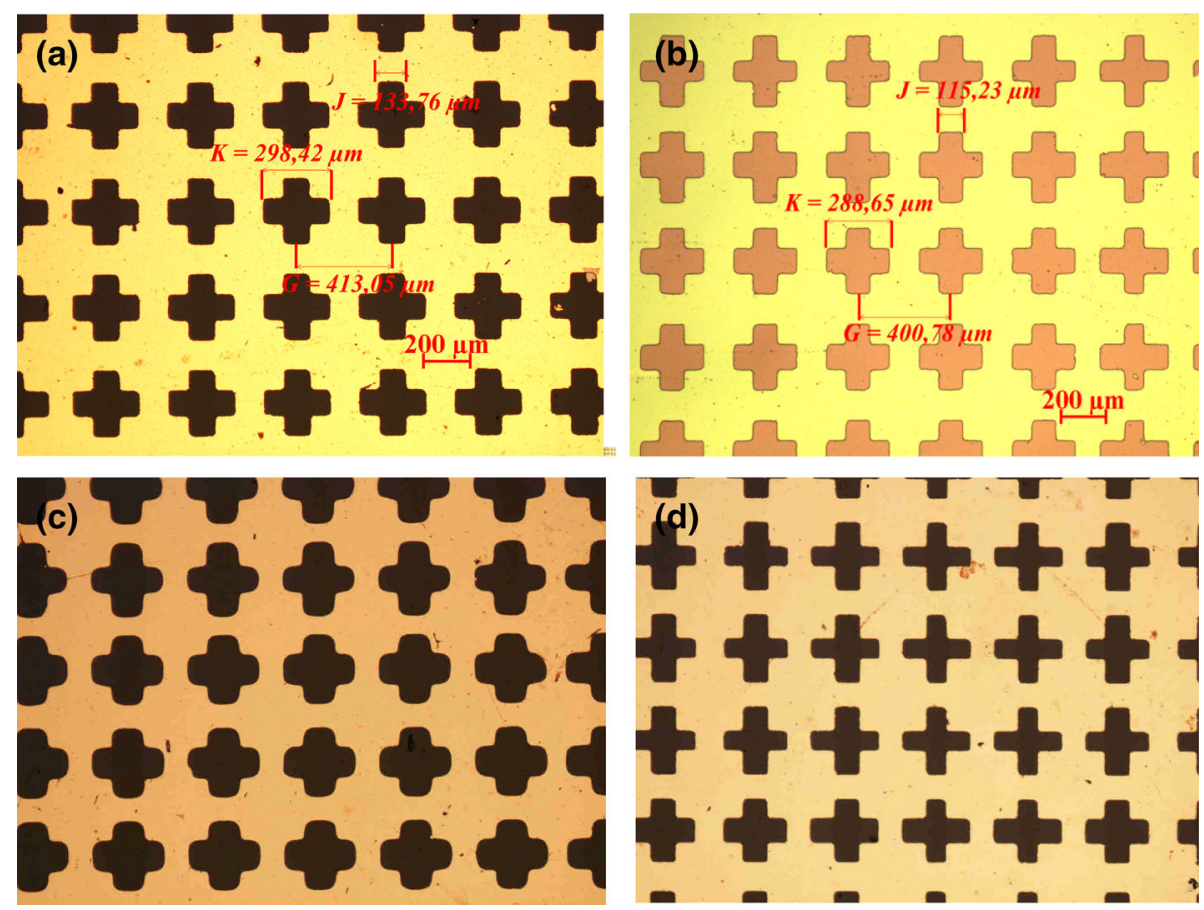

Fig. 3 a, b Optical microscope images of $\mathrm{Cu}$ and ITO metal mesh filters. c, $\mathbf{d}$ Optical microscope images of Ti and ITO metal mesh filters with local defects and also showing rounding near the edges

transmittance peak reduced as a consequence of reduction in film conductivity. With the identical mesh parameters, the simulated ITO conventional cross-shaped filter design shows a pass band at $0.340 \mathrm{THz}$ and gives a transmission peak of $24 \%$. From freestanding structure at $0.582 \mathrm{THz}$ to fabricated structure on fused silica at $0.330 \mathrm{THz}, \mathrm{a}$ resonant frequency shift of $24 \%$ given by $\lambda_{\mathrm{r}}=\lambda_{\mathrm{r} 0} \sqrt{\left(\mathrm{n}_{1}^{2}+\mathrm{n}_{2}^{2}\right) / 2}$ was obtained for the investigated metal mesh structures. Simulations show that our conventional metal mesh filters produce the expected filter responses at desired frequencies.

In the experimental procedure, thin film deposition, photolithography, and ion beam etching techniques were used for the microfabrication of the metal mesh filters. Titanium and copper thin films were grown in high vacuum dc magnetron sputtering system on fused silica substrates by a system identical to ref. (Yazici et al. 2015). We have deposited the films under identical growth conditions such as argon partial pressure, dc power and the deposition time. After a base pressure below $4.5 \times 10^{-6}$ Torr was reached, the sputtering process was started. Operating pressure was maintained at $2.5 \times 10^{-3}$ Torr with Ar gas (99.99\% purity), and target-to-substrate distance was fixed at $8 \mathrm{~cm}$. Substrates typically used to fabricate filter structures are insulators and essentially lossless at $\mathrm{THz}$ frequencies. As distinct from titanium and copper, the main feature of indium tin oxide is the combination of electrical conductivity and optical transparency in visible range which is grown by a sputtering system on fused silica substrates given in ref. (Koseoglu et al. 2015). Thick films cannot be etched due to erosion of photoresist during the etching process. The samples in this study represent the highest thicknesses and lowest surface resistances that can be reached for each material. Sheet resistance of deposited films which is invariable 
Y. Demirhan et al.

Table 1 Thickness and sheet resistance values of the metal mesh filters fabricated from Ti, ITO and $\mathrm{Cu}$ thin films

\begin{tabular}{llcc}
\hline Sample & Material & Thickness $(\mathrm{nm})$ & $R_{\mathrm{s}}$ value $(\Omega / \mathrm{sq})$ \\
\hline Ti-a & $\mathrm{Ti}$ & 92 & 14.29 \\
Ti-b & $\mathrm{Ti}$ & 92 & 14.29 \\
ITO-a & ITO & 500 & 7.50 \\
ITO-b & ITO & 500 & 7.50 \\
ITO-c & ITO & 500 & 7.50 \\
ITO-d & ITO & 500 & 7.50 \\
Cu-a & $\mathrm{Cu}$ & 370 & 0.25 \\
Cu-b & $\mathrm{Cu}$ & 370 & 0.25 \\
Cu-c & $\mathrm{Cu}$ & 350 & 0.37 \\
Cu-d & $\mathrm{Cu}$ & 350 & 0.37 \\
Cu-e & $\mathrm{Cu}$ & 350 & 0.37 \\
Cu-f & $\mathrm{Cu}$ & 350 & 0.37 \\
\hline
\end{tabular}

with film dimensions were measured using a four-terminal sensing measurement. Table 1 shows the thickness and sheet resistivity values of the samples. After the thin film deposition, THz resonant metal-mesh filters were fabricated using the UV photolithography and Ar ion beam etching techniques. During the UV lithography process, different imperfections could appear as irregularity in shape rounding etc. Figure 3 shows the optical photographs of the cross-shaped filters fabricated using Ti, ITO and $\mathrm{Cu}$ films.

Metal mesh structure of the Ti filter is showing rounding near the corners as seen in Fig. $3 c$ on the other hand we see a nearly perfect structure of ITO filter in Fig. 3d. These variations will affect the spectral properties of the $\mathrm{THz}$ filters.

\section{THz characterization methods}

The transmission characteristics of the designed $\mathrm{THz}$ bandpass metal mesh filters was analyzed by using a standard Fourier transform infrared (FTIR) spectrometer and a homebuilt terahertz time-domain spectroscopy (THz-TDS) system. The results of measurements are consistent with that of simulation by the commercial software package CST Microwave Studio in terms of predicted center frequency and bandwidth. A Bruker Vertex 80v FTIR spectrometer with DTGS detector using Mylar beam splitters was used. Measurement chamber was evacuated below $3 \mathrm{hPa}$ during both background and sample measurements and resolution was $2 \mathrm{~cm}^{-1}$. The plane of mesh filters were aligned normal to the incident radiation during FTIR measurements.

The transmission profile of the bandpass filters were also analyzed by a THz-TDS system which is driven by a Ti: $\mathrm{Al}_{2} \mathrm{O}_{3}$ mode-lock laser. A beam splitter splits the incoming beam into the two optical arms of the spectrometer; generation arm and detection arm. In the generation arm, an s-polarized terahertz beam is generated using a multi-dipole photoconductive antenna. Generated $\mathrm{THz}$ rays in antenna are guided through the system using two off-axis parabolic mirrors and two TPX lenses. THz beam is focused onto the sample with a spot diameter of about $5 \mathrm{~mm}$. In the detection arm, the $\mathrm{THz}$ pulse is measured using electro-optic detection method through a balanced photodiode. Phase-sensitive detection is done by using a lock-in amplifier and the data is recorded with the aid of a PC and data acquisition software. The frequency data is obtained with $40 \mathrm{GHz}$ resolution. 


\section{Results and discussion}

\subsection{Fourier transform infrared spectrometer measurements}

Figure 4 presents the transmission profiles for four different filter samples fabricated from $\mathrm{Ti}$, ITO and $\mathrm{Cu}$ thin films. For clarity, only a subset of samples are shown in this figure. As expected, center bandpass frequency is the same for Ti, ITO and $\mathrm{Cu}$ filters designed with same mesh parameters and is approximately around $0.33 \mathrm{THz}$. Another remarkable result is, it is seen clearly that the transmittance of the bandpass filters increases with increasing conductivity. The measured peak transmission through a Ti filter is only $15 \%$. This decrease is understandable and related to the poor conductivity of Ti films. Transmission of the ITO filters is about $24 \%$ and can be enhanced by doping of ITO thin films. Doping rate is critical for the electrical properties of ITO thin films. There is a sound correlation between the sheet resistances of the ITO thin films and the amount of oxygen vacancies and microstructure. In the ionization process, at most two free electrons per vacancy to the donor level are generated by the oxygen vacancies which gives rise to an increase in conductivity and decrease in sheet resistances $\left(\mathrm{R}_{\mathrm{s}}\right)$. The relinquishing of some oxygen atoms from the surface of the ITO thin films and the absence of free oxygen in vacuum lead to the decrease in $R_{s}$ which can be ascribed to the amendment in the crystallinity and enhancement of oxygen vacancies. Improvement of the electrical conductivity of ITO thin films provides higher transmittance of band pass filters for $\mathrm{THz}$ waves.

In addition to this, it has been shown recently that the spectral response of metal mesh filters is sensitive to local imperfections due to the fabrication process and individual variation of each mesh parameter $\mathrm{G} / \mathrm{K} / \mathrm{J}$ which can shift the peak frequency and bandwidth in a linear or non linear way (Melo et al. 2012). We have obtained similar behavior due to the over UV exposure in our filter structures as seen in Fig. 3a.

In Fig. 5, the transmission characteristics of all copper based filters having the same mesh parameters are given. The variations in the spectral data are related to the rounding of

Fig. 4 FTIR measurements of $\mathrm{Ti}$, ITO and $\mathrm{Cu}$ metal mesh filters on the same graph

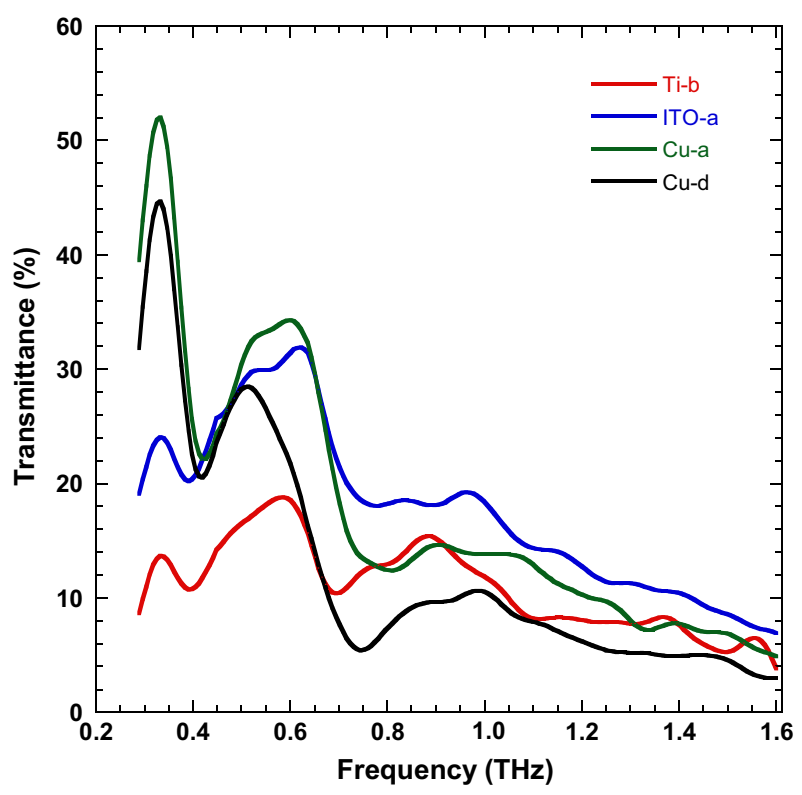




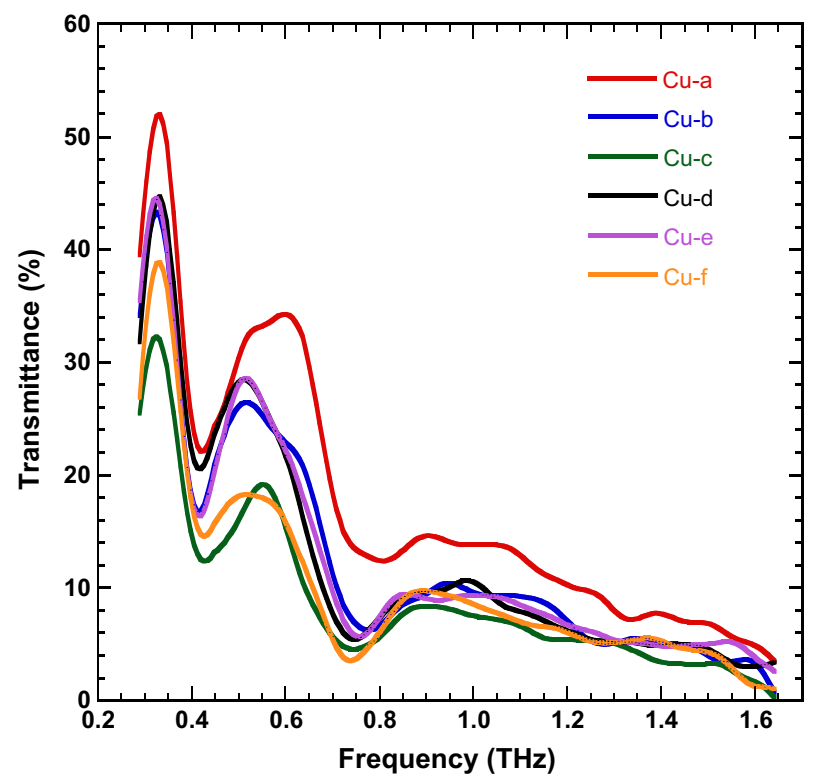

Fig. 5 FTIR measurements of all $\mathrm{Cu}$ metal mesh filters on the same graph

corners, defects and imperfections. The bandwidths were calculated to vary between 13 and $18 \%$ of the central frequency as expected since Porterfield filters typically have a bandpass width of about $15 \%$ of the central frequency. Besides, out-of-band rejection of measured cross-shaped filters is weak, as seen in Fig. 5. A way of improving the out-ofband rejection is to incorporate an inner cross to the design, causing a trapped-mode excitation, which has the opposite surface current to the outer cross structure (Paul et al. 2009). For bandwidth reduction, these filters can be fabricated on both sides of the substrate and multiple filters may be used together to narrow the transmitted spectrum for applications.

\subsection{Terahertz time-domain spectroscopy measurements}

The time-domain terahertz spectrometer employed typically has a bandwidth of about $3 \mathrm{THz}$. Due to the slight absorption profile of the fused silica substrate the usable bandwidth of the system during the measurements was limited to $1 \mathrm{THz}$. Also the TPX lenses that were used focused the beam to a diameter of about $5 \mathrm{~mm}$ on the substrate. The measurements were obtained in a dry nitrogen purged atmosphere. As can be seen in Fig. 6, the THz transmission shows a clear peak that coincides with the peak as simulated using CST Microwave Studio and shown in Fig. 2. Furthermore the broad peak at $0.5 \mathrm{THz}$ that can be attributed to the substrate is also visible, and much less in amplitude again as predicted in the simulation. Additional measurements were performed on the same pattern based on ITO as the conductive mesh layer. As can be seen in Fig. 7, the main peak is less than the transmission through $\mathrm{Cu}$ filters due to the smaller conductivity of the ITO film. The broad peak near $0.6 \mathrm{THz}$ becomes more pronounced due to the decrease in the main peak, which is similar to the case as observed with FTIR measurement for the same sample as shown in Fig. 4. The discrepancy in the peak $\mathrm{THz}$ transmission intensities near $0.3 \mathrm{THz}$ 
Fig. $6 \mathrm{THz}$ time-domain measurements of all $\mathrm{Cu}$ filters on the same graph
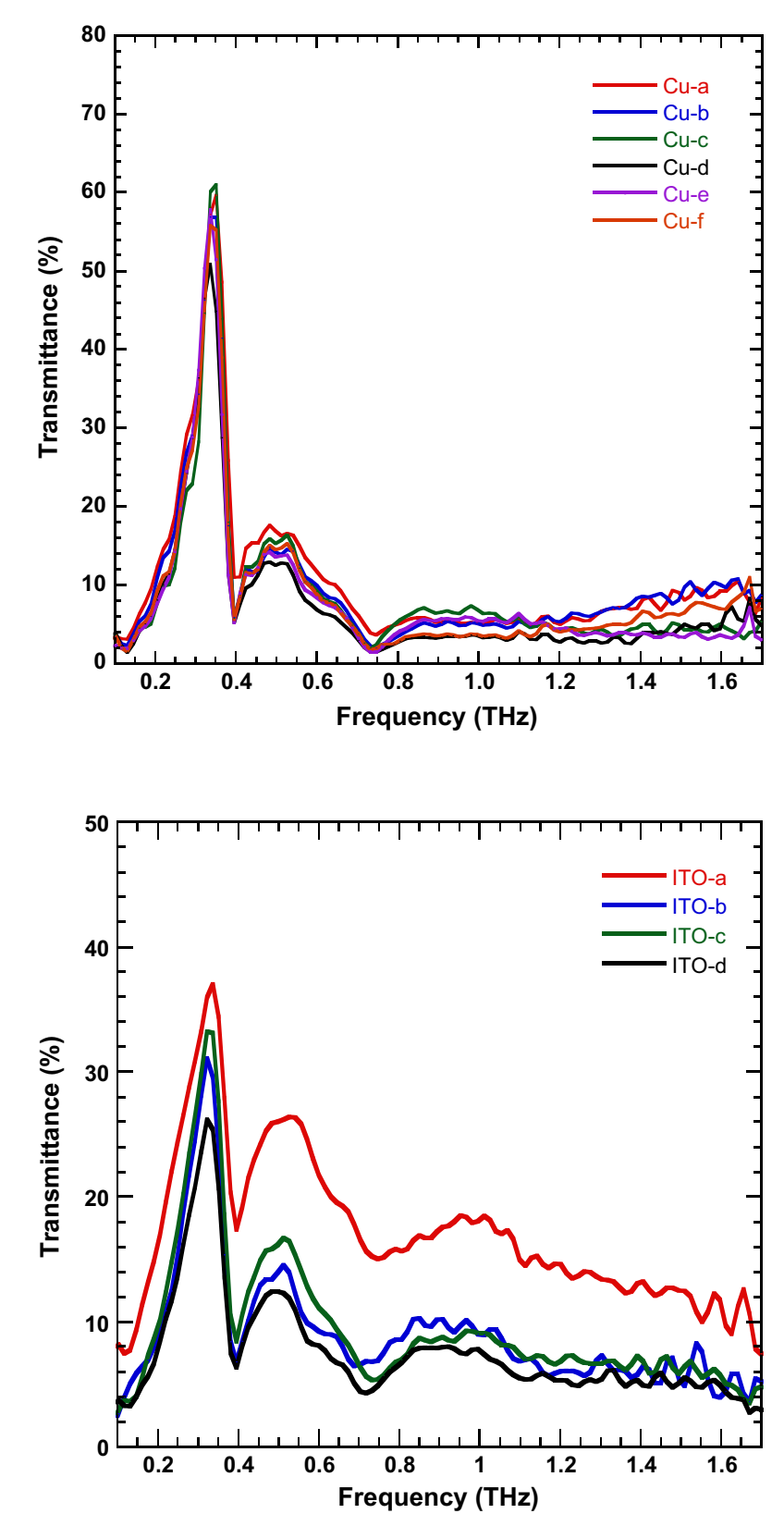

Fig. $7 \mathrm{THz}$ time-domain measurements of all ITO filters on the same graph

between the THz-TDS and FTIR measurements are most likely due to sample placement along the THz beam path. The thick fused silica substrates the films were patterned on can cause shifts in the focus of the $\mathrm{THz}$ beam which can enhance or reduce the $\mathrm{THz}$ transmission. Overall, these measurements show that THz-TDS methods are highly sensitive and can be used to characterize the response of various filters in phase as well as in amplitude of the propagated field. 


\section{Conclusion}

In summary, we have presented $\mathrm{Ti}$, ITO and $\mathrm{Cu}$ bandpass filters in the $\mathrm{THz}$ frequency range. As expected, the transmittance of the bandpass filters increases with increasing conductivity. The obtained experimental data are consistent with the simulations and observed transmission properties which reveals that these characterization methods can be utilized in the design of compact, tunable, THz filters. An insertion loss as seen in the measurements is due to the finite conductivity of the metal films and some loss in the substrate. Since there exists a $350 \mathrm{GHz}$ atmospheric transmission window, ITO coated filter structures with $330 \mathrm{GHz}$ resonant frequency are promising for future wireless $\mathrm{THz}$ communication applications. Metal mesh filters can be used to remove unwanted transmitted signals in specified frequency bands and have potential applications in the field of $\mathrm{THz}$ diagnostics and $\mathrm{THz}$ communications.

Acknowledgments This work has been supported in part by TUBITAK (Scientific and Technological Research Council of Turkey) under project number 114F091. The infrastructure of Applied Quantum Research Center (UKAM) was used to perform some parts of the experiments.

\section{References}

Ade, P.A.R., Pisano, G., Tucker, C., Weaver, S.: A review of metal mesh filters. Proc. SPIE 6275, 62750U1-62750U15 (2006)

Demirhan, Y., Saglam, H., Turkoglu, F., Alaboz, H., Ozyuzer, L., Miyakawa, N., Kadowaki, K.: Area dependence and influence of crystal inhomogeneity on superconducting properties of Bi2212 mesa structures. Vacuum 120, 89-94 (2015)

Du, J., Hellicar, A.D., Li, L., Hanham, S.M., Nikolic, N., Macfarlane, J.C., et al.: Terahertz imaging using a high- $\mathrm{T}_{\mathrm{c}}$ superconducting Josephson junction detector. Supercond. Sci. Technol. 21, 125025 (2008)

Ebbesen, T.W., Lezec, H.J., Ghaemi, H.F., Thio, T., Wolff, P.A.: Extraordinary optical transmission through subwavelength hole arrays. Nature 391, 667-669 (1998)

Ferguson, B., Zhang, X.C.: Materials for terahertz science and technology. Nat. Mater. 1, 26-33 (2006)

$\mathrm{Hu}$, Q., Richards, P.L.: Design analysis of a high $\mathrm{T}_{\mathrm{c}}$ superconducting microbolometer. Appl. Phys. Lett. 55, 2444-2446 (1989)

Kadowaki, K., Tsujimoto, M., Yamaki, K., Yamamoto, T., Kashiwagi, T., Minami, H., Tachiki, M., Klemm, R.A.: Evidence for a dual-source mechanism of terahertz radiation from rectangular mesas of single crystalline $\mathrm{Bi}_{2} \mathrm{Sr}_{2} \mathrm{CaCu}_{2} \mathrm{O}_{8+\delta}$ intrinsic josephson junctions. J. Phys. Soc. Jpn. 79, 023703 (2010)

Kaufmann, P., Marcon, R., Marun A., et al.: Selective spectral detection of continuum terahertz radiation. In: Millimeter, Submillimeter, and Far-Infrared Detectors and Instrumentation for Astronomy V, vol. 7741 of Proceedings of SPIE, San Diego, Calif, USA, July (2010)

Kimand, D.H., Choi, J.I.: Design of a multiband frequency selective surface. ETRI J. 28(4), 506-508 (2006)

Kleine-Ostmann, T., Nagatsuma, T.: A review on terahertz communications research. J. Infrared Millim. Terahertz. Waves 32, 143-171 (2011)

Koseoglu, H., Turkoglu, F., Kurt, M., Yaman, M.D., Akca, F., Aygun, G., Ozyuzer, L.: Improvement of optical and electrical properties of ITO thin films by electro-annealing. Vacuum 120, 8-13 (2015)

Ma, Y., Khalid, A., Drysdale, T.D., Cumming, D.R.S.: Direct fabrication of terahertz optical devices on lowabsorption polymer substrates. Opt. Lett. 34(10), 1555-1557 (2009)

Martin-Moreno, L., Garcia-Vidal, F.J., Lezec, H.J., Pellerin, K.M., Thio, T., Pendry, J.B., Ebbesen, T.W.: Theory of extraordinary optical transmission through subwavelength hole arrays. Phys. Rev. Lett. 86(6), 1114-1117 (2001)

Melo, A.M., Kornberg, M.A., Kaufmann, P., et al.: Metal mesh resonant filters for terahertz frequencies. Appl. Opt. 47, 6064-6069 (2008)

Melo, A.M., Gobbi, A.L., Piazzetta, M.H., Da, Silva A.M.: Cross-shaped terahertz metal mesh filters. Historical review and results. Adv. Opt. Technol. 2012, 530512 (2012) 
Ozyuzer, L., Koshelev, A.E., Kurter, C., Gopalsami, N., Li, Q., Tachiki, M., Kadowaki, K., Tamamoto, T., Minami, H., Yamaguchi, H., Tachiki, T., Gray, K.E., Kwok, W.K., Welp, U.: Emission of coherent $\mathrm{THz}$ radiation from superconductors. Sci. 318, 1291 (2007)

Park, H.R., Park, Y.M., Kim, H.S., Kyoung, J.S., Seo, M.A., Park, D.J., Ahn, Y.H., Ahn, K.J., Kim, D.S.: Terahertz nanoresonators: giant field enhancement and ultrabroadband performance. Appl. Phys. Lett. 96, 121106 (2010)

Paul, O., Beigang, R., Rahm, M.: Highly selective terahertz bandpass filters based on trapped mode excitation. Opt. Express 17, 18590-18595 (2009)

Porterfield, D.W., Hesler, J.L., Densing, R., Mueller, E.R., Crowe, T.W., Weikle, R.M.: Resonant metalmesh bandpass filters for the far infrared. Appl. Opt. 33(25), 6046-6052 (1994)

Rice, J.P., Grossman, E.N., Rudman, D.A.: Antenna-coupled high-Tc air-bridge microbolometers on silicon. Appl. Phys. Lett. 65, 773-775 (1994)

Siegel, P.H.: Terahertz technology in biology and medicine. IEEE Trans. Microw. Theory Tech. 52, 2438-2447 (2004)

Tarasov, V.D., Gromov, G.D., Bogomolov, E.A., Otto, L.S.: Fabrication and characteristics of mesh bandpass filters. Kuzmin. Instrum. Exp. Tech. 52, 74-78 (2009)

Takan, T., Keskin, H., Altan, H.: Low-cost bandpass filter for terahertz applications. Opt. Quant. Electron. 47, 953-960 (2015)

Tonouchi, M.: Cutting-edge terahertz technology. Nat. Photonics 1, 97-105 (2007)

Turkoglu, F., Ozyuzer, L., Koseoglu, H., Demirhan, Y., Preu, S., Malzer, S., Simsek, Y., Wang, H.B., Muller, P.: Emission of the $\mathrm{THz}$ waves from large area mesas of superconducting $\mathrm{Bi}_{2} \mathrm{Sr}_{2} \mathrm{CaCu}_{2} \mathrm{O}_{8+\mathrm{d}}$ by the injection of spin polarized current. Physica C 491, 7 (2013)

Turkoglu, F., Koseoglu, H., Demirhan, Y., Ozyuzer, L., Preu, S., Malzer, S., Simsek, Y., Muller, P., Yamamoto, T., Kadowaki, K.: Interferometer measurements of terahertz waves from $\mathrm{Bi}_{2} \mathrm{Sr}_{2} \mathrm{CaCu}_{2-}$ $\mathrm{O}_{8+\mathrm{d}}$ mesas. Supercond. Sci. Technol. 25, 125004 (2012)

Voisiat, B., Biciunas, A., Kašalynas, I., Raciukaitis, G.: Bandpass filters for THz spectral range fabricated by laser ablation. Appl. Phys. A 104, 953-958 (2011)

Wang, H.B., Guénon, S., Yuan, J., Iishi, A., Arisawa, S., Hatano, T., Yamashita, T., Koelle, D., Kleiner, R.: Hot spots and waves in $\mathrm{Bi}_{2} \mathrm{Sr}_{2} \mathrm{CaCu}_{2} \mathrm{O}_{8}$ intrinsic Josephson junction stacks-a study by low temperature scanning laser microscopy. Phys. Rev. Lett.102, 017006 (2009)

Winnewiser, C., Lewer, F., Weinzier, J., Helm, H.: Transmission features of frequency selective surface components in the far infrared determined by terahertz time domain spectroscopy. Appl. Opt. 38, 18 (1999)

Yazici, S., Olgar, M.A., Akca, F.G., Cantas, A., Kurt, M., Aygun, G., Tarhan, E., Yanmaz, E., Ozyuzer, L.: Growth of $\mathrm{Cu}_{2} \mathrm{ZnSnS}_{4}$ absorber layer on flexible metallic substrates for thin film solar cell applications. Thin Solid Films 589, 563-573 (2015) 